\title{
Fieber, Petechien und Bauch- und Muskelschmerzen
}

Das 10-jährige türkischstämmige Mädchen wurde aus einer auswärtigen Klinik übernommen mit hohem Fieber seit 2 Wochen sowie heftigen Bauchschmerzen. Es war eine Appendektomie durchgeführt worden, mikroskopisch zeigte die Appendix eine lymphofollikuläre Invasion mit der histologischen Diagnose einer katarrhalischen Appendizitis. Intraoperativ wurde eine ausgeprägte Lymphadenitis mesenterialis mit Enteritis und Aszites beschrieben. Zu den weiterhin persistierenden Bauchschmerzen klagte das Mädchen über ausgeprägte multitope Myalgien und Arthralgien. Im Alter von 3 Jahren war eine Purpura Schönlein-Henoch (PSH) durchgemacht worden. 2 Wochen vor der Erkrankung trat eine Rachenentzündung ohne Erregernachweis auf.

\section{Aufnahmebefund}

Deutlich reduzierter Allgemeinzustand mit Fieber bis $40^{\circ} \mathrm{C}$ und heftigsten Muskelschmerzen, sodass sich das Mädchen im Bett nicht mehr umdrehen konnte. Es zeigte sich eine diffuse Schwellung der Finger und Hände. Das Abdomen war weich, der Neurostatus ohne motorische oder sensorische Auffälligkeiten. An der Haut waren im Bereich der Füße abklingende, petechiale Effloreszenzen ( $>$ Abb. 1) zu sehen, am Unterarm livedoartige Veränderungen ( $\triangleright$ Abb. 2), an den Fingern livide Verfärbungen ( $\triangleright$ Abb. $\mathbf{3}$ ) und hämorrhagische Effloreszenzen im Sinne von Mikroinfarkten an den Ellbogenstreckseiten ( $\triangleright$ Abb. 4). Der Blutdruck war mit 140/90 mmHg im Verlauf deutlich oberhalb der $95 \%$ Perzentile für altersgemäße Blutdruckwerte.

\section{Labor}

- Hb 9,1 g/dl, CRP max 24 mg/dl, BSG $>100 / 1 \mathrm{~h}$

- Creatinin, GOT, CK, Lipase, Ferritin, Creatinkinase jeweils im Normbereich

- Albumin $20 \mathrm{~g} / \mathrm{l} / \mathrm{l})$, ANA, C3, C4, MPO AK, PR-3 Ak negativ, IgA, IgG, IgM im Normbereich

- Serologie auf Influenza, Leptospiren, EBV, Bartonellen, Hantavirus, Puumalavirus und T-Spot jeweils unauffällig

- Urin 2-fach positiv auf Erythrozyten im Stix und im Sediment

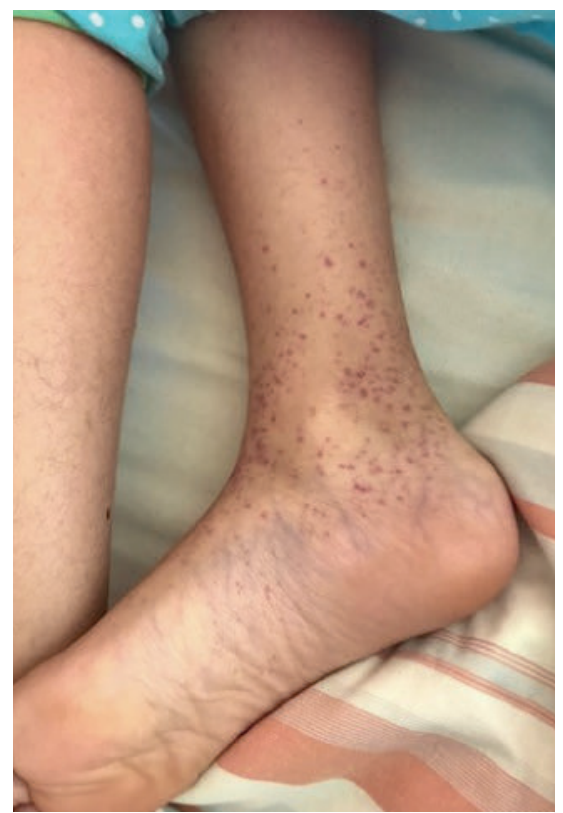

- Abb. 1 PSH-typische Effloreszenzen.

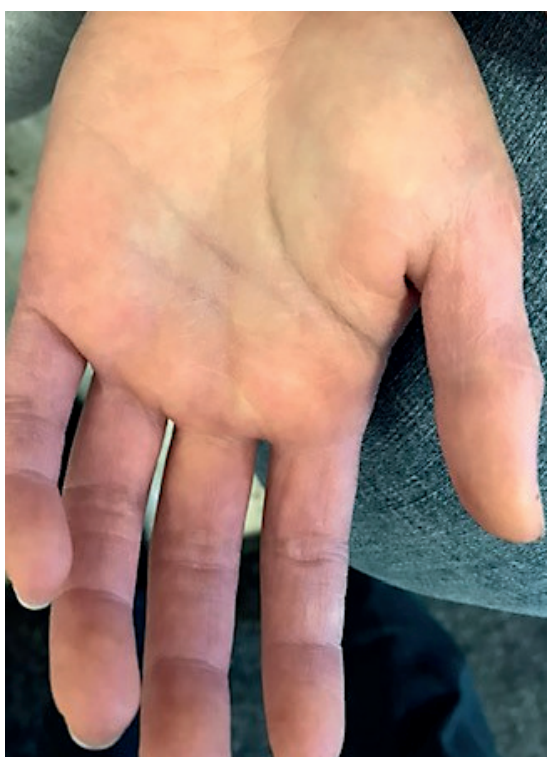

- Abb. 3 Livide Finger und schmerzhafte Schwellung der Hand.

\section{Weitere Diagnostik}

Im Röntgen-Thorax war ein diskreter Pleuraerguss zu sehen, die Sonografie des Abdomens war unauffällig. Eine Ganzkörper-MRT zeigte im Bereich der gesamten Muskulatur in der STIR-Sequenz eine deutliche Signalanhebung passend zu einer Myositis ( $\triangleright$ Abb. 5).

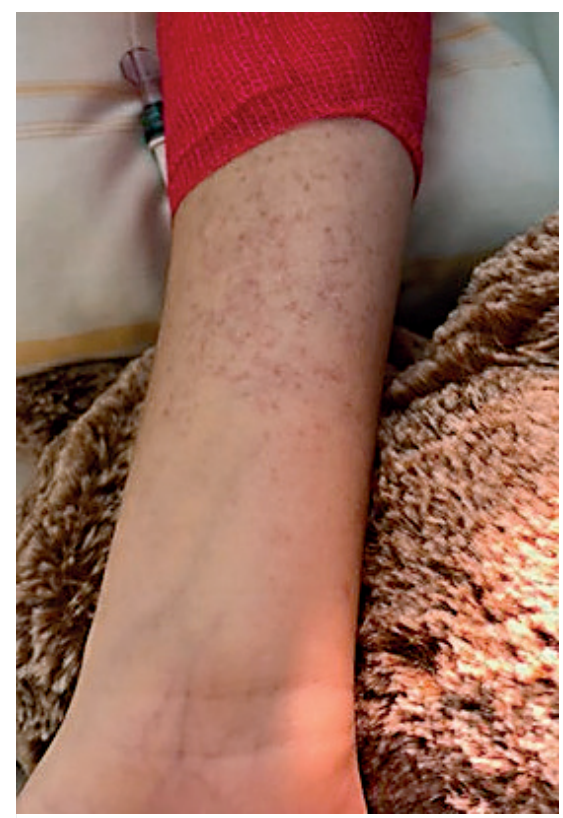

- Abb. 2 Livedo retikularis im Bereich des Unterarmes.

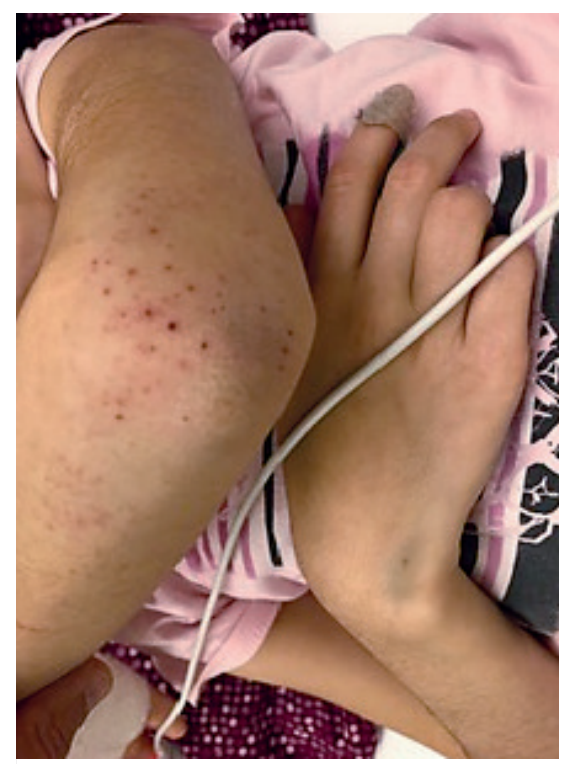

Abb. 4 Mikroinfarkte.

\section{Differenzialdiagnose}

Differenzialdiagnostisch war aufgrund der ethnischen Herkunft ein familiäres Mittelmeerfieber zu diskutieren. Allerdings beträgt die Fieberdauer hierbei maximal 5 Tage. Eine systemische juvenile Arthritis war aufgrund des fehlenden zirkadianen Fieberrhythmik und des Hautbefunds 


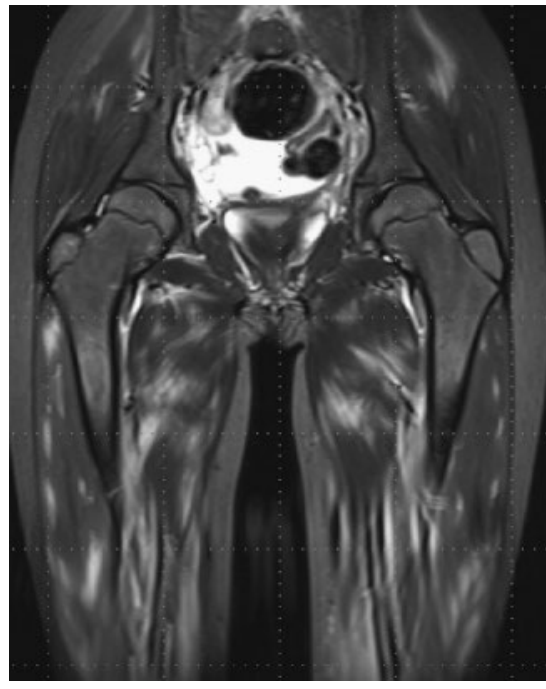

- Abb. 5 Die MRT zeigt in der STIRSequenz multiple muskuläre Signalanhebungen passend zu einer Myositis.

nicht typisch. Für eine leukozytoklastische Vaskulitis im Sinne einer PSH war das Kind zu krank.

Der Schlüssel zur Verdachtsdiagnose waren die von der Mutter fotografierten livedoartigen Effloreszenzen im Bereich der Unterarme. In der Gesamtkonstellation mit Fieber, Myalgien und Livedo retikularis wurde die Verdachtsdiagnose einer Polyarteriitis nodosa (PAN) gestellt.

\section{Bestätigungsdiagnostik}

Eine MRT-Angiografe der Abdominalgefäße zeigte einen unauffälligen Befund. Als nächster Schritt wurde eine tiefe Muskelbiopsie angeschlossen, bei der allerdings histologisch keine Arterien nachgewiesen werden konnten. Erst in der konventionellen Angiografie konnte der Nachweis von multiplen Aneurysmen in der Arteria hepatica erbracht und die Diagnose einer PAN gesichert werden ( $\triangleright$ Abb. 6).

Die nachträglich eingetroffene Molekulargenetik erbrachte eine heterozygote Mutation im MEFV-Gen (M964V), das ADA2-Gen war nicht mutiert.

\section{Diskussion}

Die PAN ist eine nekrotisierende Vaskulitis der mittleren und kleinen Arterien. Arteriolen, Kapillaren und Venen sind nicht betroffen. Eine Glomerulonephritis ist eben-

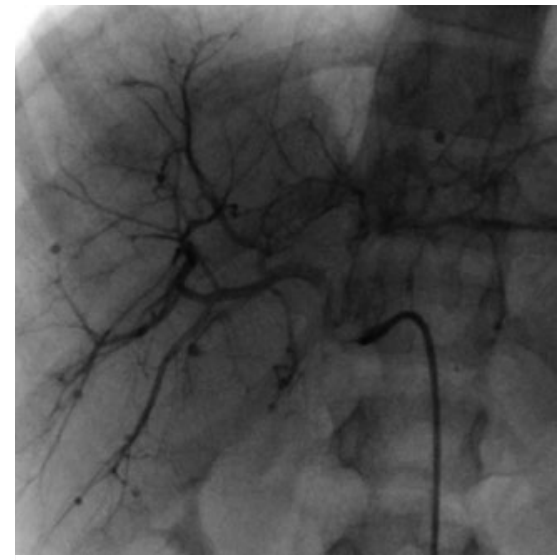

Abb. 6 Konventionelle selektive Angiografie: Nachweis multipler Aneurysmen in der Arteria hepatica und Verzweigungen.

so nicht vorhanden wie der Nachweis von antineutrophilen cytoplasmatischen Antikörpern (ANCA) [1]. Die Krankheit wurde erstmalig von Kussmaul und Maier 1866 beschrieben unter dem Titel „Über eine bisher nicht beschriebene eigenthümliche Arterienerkrankung (Periarteriitis nodosa), die mit Morbus Brightii und rapid fortschreitender allgemeiner Muskellähmung einhergeht" (Dt. Archiv für klinische Medizin, Leipzig 1866;1:484-518).

Der Hautbefund zeigte zunächst den typischen Befund einer PSH. Das Krankheitsgefühl des Mädchens mit ausgeprägten Myalgien und Bauchschmerzen sowie das hohe Entzündungslabor ließen aber an eine PAN denken. Die namensgebenden knötchenförmigen Hauteffloreszenzen treten nur bei einem geringen Teil der Patienten auf. Eine der größten Studie aus der Türkei mit 133 Patienten (darunter 66 Kinder und Jugendliche) zeigte eine Pannikulitis bei nur $27 \%$ der pädiatrischen Patienten [1]. Die häufigsten Manifestationen waren dabei Fieber (76\%), Myalgien (72\%), Bauchschmerzen (61\%), Arthralgien (73\%) und Livedo retikularis (55\%). Eine ähnliche Verteilung der klinischen Symptome wurde auch in der bislang größten Kohorte 2013 in England beschrieben [2]. In einer Übersichtsarbeit der französischen Vasculitis study group an 348 erwachsenen Patienten zeigte sich, dass Knoten nur zu $17 \%$, Purpura zu 22\% und Livedo zu 17\% vorkommen. Allgemeinsymptome wie Fieber, Gewichtsverlust, Myalgien und Arthralgien sowie eine periphere Neuropathie waren die häufigsten Symptome und Befunde [3].
Auch PSH-typische Veränderungen wurden bei der PAN beschrieben [4].

Die häufig auftretenden gastrointestinalen Symptome sind teilweise sehr heftig, sodass immer wieder Appendektomien erfolgten [5-7].

Da die bunte und eher unspezifische Symptomatik oft nicht gleich an das Krankheitsbild der PAN denken lässt, ist mit einer mittleren Diagnoseverzögerungsdauer von 7 Monaten zu rechnen [3]. Aus klinischer Sicht ist bei unklarem Fieber, schlechtem Allgemeinzustand mit Muskel- und Bauchschmerzen sowie vaskulitischen Effloreszenzen, Pannikulitis oder Knoten eine PAN zu erwägen. Die Erkrankung kann allerdings auch in lokalisierter Form auftreten mit einem isoliertem Organ- oder Extremitätenbefall $[8,9]$.

Mittlerweile ist eine genetische Variante der PAN bekannt geworden, die DADA-2-Erkrankung mit Defizienz der AdenosinDeaminase. Es wird verursacht durch eine Mutation im ADA-2-Gen. Bei DADA-2 sind neben der PAN-Symptomatik frühzeitige zerebrale Insulte charakteristisch. Jedoch ist auch hierbei die klinische Symptomatik breit und unspezifisch und keines der Symptome oder Befunde treten mit einer Häufigkeit von über $50 \%$ auf [10].

Darüber hinaus sind assoziierte genetische Mutationen im MEFV-Gen bekannt. In der Studie von Sönmez 2019 fand sich bei 24 von 65 untersuchten Patienten mindestens eine Mutation in diesem Gen [1]. Auch bei unserer Patientin war eine heterozygote Mutation an typischer Stelle (M694V) nachweisbar.

\section{Klassifikationskriterien}

Die EULAR-Kriterien von 2010 fordern zur Diagnose den histopathologischen Nachweis einer nekrotisierenden Vaskulitis oder angiografische pathologische Veränderungen plus eines der weiteren $5 \mathrm{Kri}$ terien: Hautbeteiligung, Myalgien, arterielle Hypertonie, periphere Neuropathie, Nierenbeteiligung [11] (siehe Kasten). Die in unserem Fall - in der MRT gesehene - Myositis ist in den o. g. Kriterien nicht enthalten. 
KLASSIFIKATIONSKRITERIEN DER KINDLICHEN PAN

Nachweis einer nekrotisierenden Vaskulitis der mittleren und kleinen Gefäße oder eine angiografische Auffälligkeit mit Aneurysmen, Stenosen oder Okklusion einer mittleren oder kleinkalibrigen Arterie plus eines der folgenden 5 Kriterien:

1. Hautbeteiligung (Livedo reticularis, Hautknoten oder -infarkte)

2. Myalgie oder muskulärer Druckschmerz

3. Bluthochdruck>95. Altersperzentile

4. Periphere Neuropathie (sensorisch periphere Neuropathie oder motorische Mononeuritis multiplex)

5. Nierenbeteiligung (Proteinurie $>0,3 \mathrm{~g} / 24$ Std oder $>30 \mathrm{mmol} /$ mg der Urinalbumin/CreatininRatio im Spontanurin; Hämaturie oder Erythrozytenzylinder, $>5$ Erythrozyten/High-power-Feld, Erythrozytenzylinder im Sediment oder entsprechend $2+i m$ Urinsticks; oder eingeschränkte Nierenfunktion berechnet nach der glomerulären Filtrationsrate (Schwartzsche Formel) $<50 \%$ unter dem Normalbereich

Die Diagnosesicherung kann grundsätzlich histopathologisch mittels einer Biopsie befallener Strukturen der Haut oder der Muskulatur erfolgen. Bei der Muskelbiopsie ist eine tiefe gezielte Probenentnahme zu anzuraten - z. B. über eine vorherige Lokalisation mittels MRT [12]. Dabei wird eine Muskelprobe von ca. $2,5 \mathrm{~cm}$ Länge und $0,5 \mathrm{~cm}$ Durchmesser empfohlen (persönliche Mitteilung Fr. Priv.-Doz. Dr. Holl-Ulrich, Vaskulitiszentrum Marienkrankenhaus Hamburg). Dies erhöht die Wahrscheinlichkeit, eine Arterie einzuschließen, ohne die eine Diagnose nicht möglich ist.

Ein histologisch fehlender Nachweis schließt eine PAN nicht aus, da die Verteilung von Läsionen in der Muskulatur sehr heterogen sein kann [12]. In der Studie von Sönmez 2019 hatten 109/133 Patienten eine Biopsie (der Haut bei 94, der Muskulatur bei 6, der Niere bei 9). Wie von den
Autoren erwähnt, zeigten alle eine nekrotisierende Vaskulitis. Andere Studien wiesen mit 20-35\% eine eher geringere Sensitivität auf $[12,18]$.

Der andere Hauptpfeiler der Diagnose ist der angiografische Nachweis von Aneurysmen. In den Klassifikationskriterien, wie auch in den aktuellen SHARE-Empfehlungen der europäischen Fachgesellschaft, wird die konventionelle Angiografie als Goldstandard empfohlen $[11,12]$. Die zugrunde liegenden Arbeiten beziehen sich vor allem auf die Studien von Brogan aus dem Jahre 2000 und dem erweiterten Patientenklientel von Eleftheriou aus dem Jahre 2013, die über die größte Single-Center-Kohorte mit 69 Patienten berichten [2]. Dabei wurde die konventionelle Angiografie als sensitiver im Vergleich zur Sonografie oder CT-Angiografie beschrieben. Allerdings sind keine vergleichenden Daten zwischen konventioneller Angiografie und MRT-Angiografie erwähnt. In einer anderen Studie wurde für die konventionelle Angiografie eine Sensitivität von $89 \%$ und eine Spezifität von $90 \%$ ermittelt [19]. Allerdings ist zu beachten, dass auch diese Untersuchung anfangs oder nach Steroidtherapie negativ sein kann [12]. In der Studie von Sönmez 2019 wurde bei 92/133 eine CT-Angiografie durchgeführt, dabei wurden bei 69 renale Nierenarterienaneurysmen gefunden, 17 wiesen Leberarterienaneurysmen auf.

Die konventionelle Angiografie hat somit den Vorteil einer guten Detaildarstellung kleiner Aneurysmen und ist der MRT- und CT-Angiografie in der Auflösung überlegen. Dabei ist zu beachten, dass alle intraabdominellen Gefäße (Truncus zöliacus, Art. renalis, mesenterica, hepatis) einschließlich ihrer peripheren Äste untersucht werden müssen. In einem frühen Stadium der Erkrankung oder unter Therapie kann es allerdings sein, dass Aneurysmen bildgebend nicht nachweisbar sind. Die konventionelle selektive Angiografie kann als invasives Verfahren allerdings auch mit Komplikationen behaftet sein und sollte deshalb nur von erfahrenen Untersuchern durchgeführt werden.

Neben den typischen Aneurysmen hat Brogan 2002 auch nichtaneurysmatypische Gefäßveränderungen bei der renalen
Angiografie beschrieben, wie Perfusionsdefekte, Kollateralarterien, fehlende Aufzweigung (Crossing) peripherer Nierenarterien und verzögerte Entleerung der Arterien [15]. Die Sensitivität und Spezifität des angiografischen Nachweises von Nierenarterienaneurysmen für die Diagnose PAN war $43 \%$ bzw. $69 \%$ und konnte für die Sensitivität gesteigert auf $80 \%$ werden, wenn nichtaneurysmatische Veränderungen hinzugenommen wurden. Allerdings sank dabei die Spezifität auf 50\%. Zudem wurden auch Gefäßveränderungen in den hepatischen, splenalen und viszeralen Arterien gesehen [15].

Andere Autoren empfehlen eine noninvasive Diagnostik. Özcakar berichete über 9 Patienten mit den Symptomen Bauchschmerzen, Fieber, Fatigue und Myalgien. Hierbei konnte bei 7 die Diagnose mit Dopplerultraschall und bei 4 mit zusätzlicher CT-Angiografie gestellt werden [17]. Auch wenn dazu keine vergleichende Studie existiert, ist davon auszugehen, dass die Sensitivität der jeweiligen Methode vor allem durch mangelnde räumliche Auflösung und/oder Bewegungsartefakte beeinträchtigt wird. Unter dieser Voraussetzung kann die Diagnostik mit nicht invasiven Methoden ohne Strahlenexposition wie Sonografie und MRT begonnen werden. Als sensitivste Methode zum Nachweis kleiner Aneurysmen z. B. in der atemverschieblichen Leber muss jedoch die Katheterangiografie angesehen werden ( $\triangleright$ Abb. 6 ).

\section{Therapie}

Die Therapie sieht induktiv hochdosierte Steroidgaben vor sowie Cyclophosphamidpulse bei schwerem Befall. Aufgrund des jugendlichen Alters unserer Patienten haben wir anstelle von Cyclophosphamid eine Therapie mit Mycophenolatmofetil begonnen. Mit diesem Medikament wurde unlängst in England eine Studie durchgeführt (MYPAN), deren Ergebnisse noch ausstehen [20]. Zudem haben wir eine Therapie mit Low-dose-ASS begonnen. Bei fehlendem Ansprechen kommen Biologika zum Einsatz (TNF- $\alpha$-Blocker, B-Zell-Depletion, IL-6-Blockade). Zur Dauer der Erhaltungstherapie existieren keine klaren Daten. Sie sollte aber nicht vor 12-36 Monaten in Remission beendet und dann über ca. 6 Monate ausgeschlichen werden [12]. 


\section{Interessenkonflikt}

Der korrespondierende Autor bestätigt, dass kein Interessenkonflikt besteht.

\section{Einhaltung ethischer Richtlinien}

Der korrespondierende Autor bestätigt, dass eine Genehmigung der Eltern der Patientin sowie der Patientin selbst zur Veröffentlichung dieses Fallberichts vorliegt.

\section{Autorinnen/Autoren}

Toni Hospach ${ }^{1}$, Fabian Glowatzki ${ }^{1}$, Friederike Blankenburg', Felix Noll', Christian Stirnkorb ${ }^{1}$, Damaris Hübner ${ }^{1}$, Thekla von Kalle ${ }^{2}$, Markus Nasko ${ }^{2}$, Konstanze Holl-Ulrich ${ }^{3}$, Ulrich Schweigmann ${ }^{4}$, Frank Uhlemann ${ }^{4}$

${ }^{1}$ Zentrum für pädiatrische Rheumatologie am Klinikum Stuttgart (ZEPRAS),

Olgahospital

2 Radiologisches Institut Olgahospital Klinikum Stuttgart

${ }^{3}$ Institut für Pathologie des MVZ am Marienkrankenhaus Hamburg

${ }^{4}$ Zentrum für angeborene Herzfehler Olgahospital, Klinikum Stuttgart

\section{Korrespondenzadresse}

\section{Dr. med. Toni Hospach}

Zentrum für Pädiatrische Rheumatologie am Klinikum Stuttgart (ZEPRAS), Klinikum Stuttgart gKAöR, Olgahospital, Pädiatrie 2 Kriegsbergstr. 62, 70174 Stuttgart A.Hospach@klinikum-stuttgart.de

\section{Literatur}

[1] Sonmez HE, Berkan A, Ayanet G et al. Polyarteritis nodosa: lessons from 25 years of experience. Clin Exp Rheumatol 2019; 37 Suppl 117(2): 52-56
[2] Eleftheriou D, Dillon M], Tullus K et al. Systemic polyarteritis nodosa in the young: a single-center experience over thirty-two years. Arthritis Rheum 2013; 65(9): 2476-2485

[3] Pagnoux C, Seror R, Henegaret C et al. Clinical features and outcomes in 348 patients with polyarteritis nodosa: a systematic retrospective study of patients diagnosed between 1963 and 2005 and entered into the French Vasculitis Study Group Database. Arthritis Rheum 2010; 62(2): 616-626

[4] Braungart S, Campbell A, Besarovic S. Atypical Henoch-Schonlein purpura? Consider polyarteritis nodosa! BMJ Case Rep 2014; 2014

[5] Starobinska E, Sekhon K, Bilal J. Abdominal pain leading to incidental finding of polyarteritis nodosa. BMJ Case Rep 2018; 2018

[6] Ozcakar ZB, Yalçinkaya F, Fitoz S et al. Polyarteritis nodosa: successful diagnostic imaging utilizing pulsed and color Doppler ultrasonography and computed tomography angiography. Eur J Pediatr 2006; 165(2): 120-123

[7] Corbitt M, Kurtkoti ], Rashid M et al. Polyarteritis nodosa presenting atypically as appendicitis and pyelonephritis et al. Polyarteritis nodosa presenting atypically as appendicitis and pyelonephritis in a single patient. BMJ Case Rep 2019; 12(2)

[8] Hall C, Mongey AB. Unusual presentation of polyarteritis nodosa. J Rheumatol 2001; 28(4): 871-873

[9] de Vries W, Koppelman GH, Roofthooft MTR et al. Pulmonary medium vessel vasculitis in an 11 year old boy: Hughes Stovin syndrome as a variant of polyarteritis nodosa? Pediatr Rheumatol Online J 2011; 9: 19

[10] Meyts I, Aksentijevich I. Deficiency of Adenosine Deaminase 2 (DADA2): Updates on the Phenotype, Genetics, Pathogenesis, and Treatment. J Clin Immunol 2018; 38(5): 569-578

[11] Ozen S, Pistorio A, lusan SM et al. EULAR/ PRINTO/PRES criteria for Henoch-Schonlein purpura, childhood polyarteritis nodosa, childhood Wegener granulomatosis and childhood Takayasu arteritis: Ankara 2008. Part II: Final classification criteria. Ann Rheum Dis, 2010. 69(5): p. 798-806
[12] de Graeff N, Groot N, Brogan P et al. European consensus-based recommendations for the diagnosis and treatment of rare paediatric vasculitides - the SHARE initiative. Rheumatology (Oxford) 2019; 58(4): 656-671

[13] Eleftheriou D, Cox T, Saunders D et al. Investigation of childhood central nervous system vasculitis: magnetic resonance angiography versus catheter cerebral angiography. Dev Med Child Neurol 2010; 52(9): 863-867

[14] de Graeff N, Groot N, Brogan P et al. European consensus-based recommendations for the diagnosis and treatment of Kawasaki disease - the SHARE initiative. Rheumatology (Oxford) 2019; 58(4): 672-682

[15] Brogan PA, Davies R, Gordon I et al. Renal angiography in children with polyarteritis nodosa. Pediatr Nephrol 2002; 17(4): 277-283

[16] Miller DL. Angiography in polyarteritis nodosa. AJR Am J Roentgenol 2000; 175(6): 1747-1748

[17] Ozcakar ZB, Fitöz S, Yildis AE et al. Childhood polyarteritis nodosa: diagnosis with non-invasive imaging techniques. Clin Rheumatol 2017; 36(1): 165-171

[18] Bron KM, Strott CA, Shapiro AP. The Diagnostic Value of Angiographic Observations in Polyarteritis Nodosa. A Case of Multiple Aneurysms in the Visceral Organs. Arch Intern Med 1965; 116: 450-454

[19] Hekali P, Kajander H, Pajariet R et al. Diagnostic significance of angiographically observed visceral aneurysms with regard to polyarteritis nodosa. Acta Radiol 1991; 32(2): 143-148

[20] Hampson LV, Whitehead ], Eleftheriou D et al. Elicitation of expert prior opinion: application to the MYPAN trial in childhood polyarteritis nodosa. PLoS One 2015; 10(3): e0120981

\section{Bibliografie}

DOI https://doi.org/10.1055/a-1214-4526 arthritis + rheuma 2020; 40: 270-273 (c) Georg Thieme Verlag KG Stuttgart . New York ISSN 0176-5167 